\title{
Mood based Playlist Generation for Hindi Popular Music: A Proposed Model
}

\author{
Kunjal Gajjar \\ Faculty, SCS, AU \\ Ahmedabad -380009 \\ Gujarat, India
}

\author{
Siddhi Shah \\ Faculty, SCS, AU \\ Ahmedabad -380009 \\ Gujarat, India
}

\begin{abstract}
Large digital databases of Hindi music are available which creates an opportunity of filtering this data with multiple parameters. One of the most important parameter used by the listeners are their moods. This paper focuses on Automatic generation of mood based playlist for Hindi popular music with minimum user intervention. There are two major modules of the proposed system. The first module identifies user's mood based on the inputs from social media and messaging app like WhatsApp. The second module is responsible for tagging the songs of available database. Tagging is done on the basis of Genre, Artists, Tempo and Lyrics. Using the above mentioned modules, mood based playlist can be generated for user.
\end{abstract}

\section{General Terms}

Playlist generation, mood analysis, meta-data analysis

\section{Keywords}

Music Information Retrieval, Mood Classification, genre, lyrics, tempo analysis, social media

\section{INTRODUCTION}

The size of multimedia data on the web is increasing in the exponential way and it provides easy accessibility to all kind of music. Due to this increase in data a new research area of Music Information Retrieval has emerged. . It deals with retrieval of data from large database by searching the content and finding the pattern rather than just using limited information available in Metadata. This has made searching of audio data more efficient. Also there are multiple approaches made for creation of playlist based on user preferences. With the advancement in the algorithms based on searching data from the large digital corpus, users are now interested in filtering the data based on various properties such as year, lyrics, genre, rhythm etc.

Now a day music has become a part of human life. In the Indian context, people relate song to any occasion or situation. Indian music has a rich history and large dataset of songs for every mood. If we narrow down to the Hindi popular music still it can be categorized under specific mood or list of moods. Indian music industry is built with the contribution from pop music industry as well as from the Bollywood industry. Hence the corpus is quite large compared to other music industries in the world. Increase in online music libraries has expanded approaches for selecting the songs and has also made it complex as user might get confused or lost in the large dataset. Further it can also create a situation of long tailing.

Because of the complexity of Hindi popular music, any one parameter such as metadata, rhythm or lyrics cannot be considered for classification of song according to the mood category. The proposed model considers combination of multiple parameters to precisely identify the mood of song.

The common property used by people for playlist generation is their mood. In current systems the user have to select songs based on their mood manually. The automated system will be useful in increasing the speed to generate playlist.

\section{EXISTING WORK}

The paper focuses on mood classification also known as emotion classification based on the lyrics. They have developed a machine learning approach to categorize song based on mood. The mood which are authors have considered for classification are identified first and then features of song are extracted for categorizing it into mood class. In their approach they have considered term frequency and inverse document frequency methods for computation [2].

The author has considered the Metadata like Artist name, Album, Genre, Tempo and Track name for categorizing the song. A hierarchical structure is built for the algorithm which focuses on the information extracted from the metadata and categorizes the song accordingly [3].

This paper concentrates only on Modern Greek musical data. It uses the approach of lyrics as well as acoustic signal features to identify mood of the song. The authors have used multiple algorithms and preprocessing parameter to generate mood model of the song [4].

Authors have presented a hierarchical framework to automate the task of mood detection from acoustic music data. They have considered intensity, timbre and rhythm to classify mood in western culture [8].

The authors have proposed a prototype for software tool for automatic generation of mood based playlist. They have considered the Thayer plane and proposed a model for generating playlist based on one seed song or based on the trajectory path drawn by the user. The application is developed using Qt framework, a C++ application and UI development framework. It also uses the Marsyas framework as part of the feature extraction logic, as well as the supervised learning algorithms offered by the libSVM3 library for mood prediction [9].

The authors have identified the 5 moods from popular Indian music for classification of song. They have followed the approach of extracting audio and lyrics features and used them in mood identification module. The mood they have used for classification are: Happy, Sad, Silent, Excited and Romantic, as these are the most used moods for Indian popular music. For implementation of their model they have used jAudio and Marsyas open source tools for extracting selective timbral, temporal and rhythm related audio features from the music pieces [11] [14]. 
Authors have developed a feature extraction framework: JAudio. This system helps the MIR researchers by eliminating the duplicate efforts of feature extraction as it provides the library of algorithms which can be used for MIR research. There are 27 features implemented in JAudio. Beat Histogram is one of the feature for representing rhythmic regularities. This feature can be used for identifying the tempo of a song [12].

Using a machine learning approach, authors have discovered association between songs and their lyrics using feeling classification method. They also discovered the common characteristics between music and lyrics [15].

The authors have developed a table related to top ranked content word features for moods. They have derived user generated tags form last.fm and identified 18 mood categories which fit well with russell's mood model [17].

\section{PROPOSED MODEL}

The proposed model is responsible for creating a mood based playlist by matching the user's mood with the tag of songs. For this model authors have considered predefined mood categories such as Happy, Sad, Excited, Romantic and Trance for tagging the songs as well as identifying the mood of user.

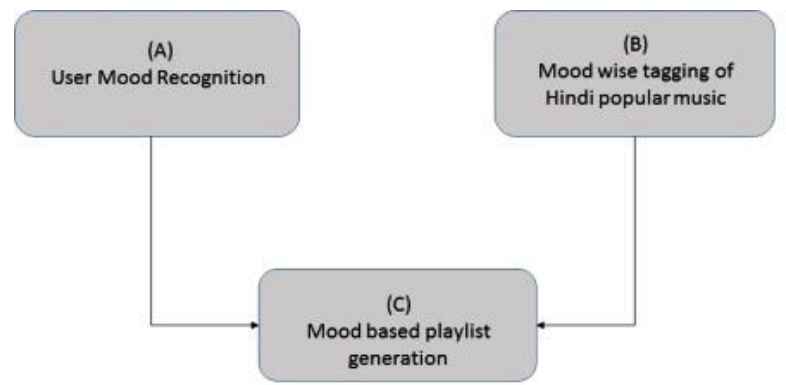

Fig 1: Model for mood based playlist generation

As shown in Fig 1, proposed model has three sub modules: User Mood Recognition, Mood wise tagging of Hindi popular music, Mood based playlist generation. Module A will identify user's mood and Module B will tag the songs according the category of mood it falls in. Playlist generation algorithm will use output from both the modules and match the tags with the identified mood of the user.

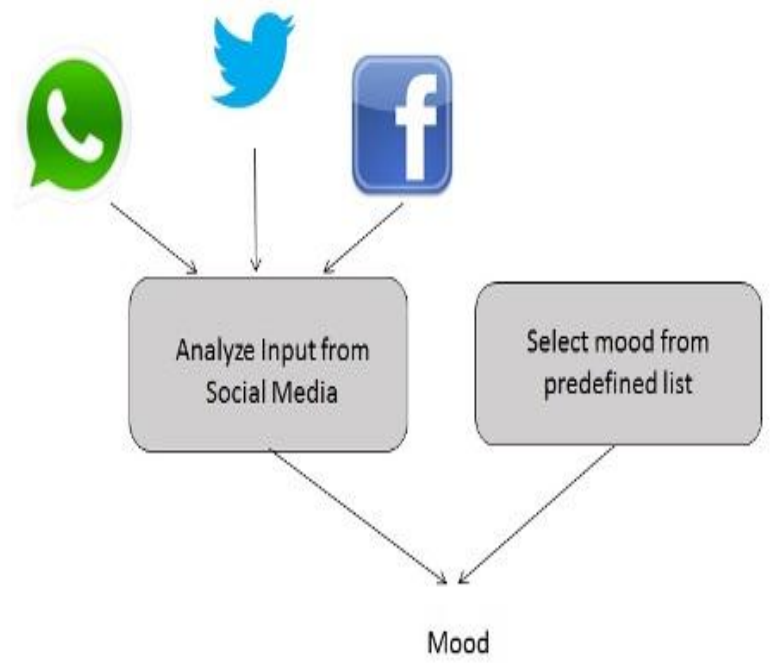

Fig 2: User's mood identification from social media and messaging app
Mood recognition module takes the input from various social media sites and messaging app like Facebook posts, Twitter tweets and WhatsApp status. The data received from these sources is analyzed by using algorithms of natural language processing for understanding the meaning of posts, tweets and status. The output of analysis is the mood identification the user without direct interaction with user. For users not using social media or interested in choosing mood manually, the list of predefined moods is also available. Model is graphically represented in Fig 2.

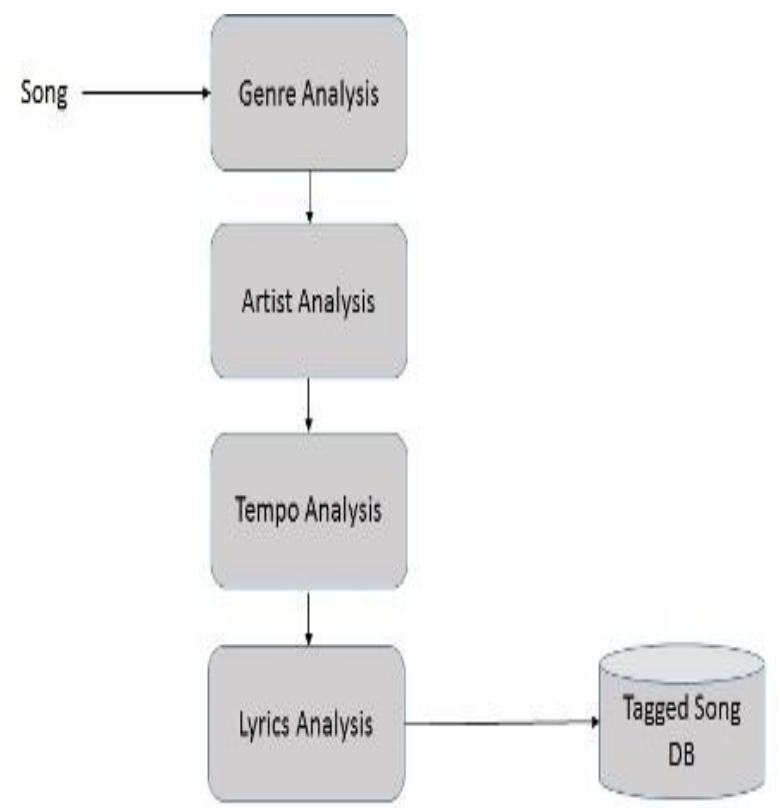

Fig 3: Mood wise tagging of songs in Database (DB)

This module (Fig 3 ) is responsible for tagging the songs in the database to classify them into various mood categories. The first sub module for tagging is genre analysis which uses the metadata information of the song. Based on the genre analysis, the songs can be classified into mood categories at the basic level. Through the analysis done from various music portals and libraries the common genre of Hindi popular music can be categorized as shown in table 1. After genre analysis the subset of mood is decided for a song.

Table 1. Genre based mood classification

\begin{tabular}{|l|l|}
\hline \multicolumn{1}{|c|}{ Genre } & \multicolumn{1}{c|}{ Mood } \\
\hline Pop & Happy, Excited \\
\hline Devotional & Sad, Happy,Trance \\
\hline Gazal & Romantic, Sad \\
\hline Sufi & Trance, Sad \\
\hline Rock & Excited,Happy \\
\hline Bollywood & Any mood \\
\hline
\end{tabular}

The second module further helps for specific mood identification based on the artist details which is available as part of metadata. In case of multiple artist in a song, categories for both the artist can be chosen. 
Table 2. Artist based mood classification

\begin{tabular}{|c|c|}
\hline Singer & Mood \\
\hline Lata Mangeshkar & Romantic, Sad, Happy \\
\hline Asha Bhosle & Excited, Happy, Sad \\
\hline Kishore Kumar & Romantic, Happy, Excited \\
\hline Mukesh Mathur & Sad, Trance \\
\hline Mohamad Rafi & Romantic, Sad, Happy \\
\hline Mika Singh & Excited \\
\hline Honey Singh & Excited \\
\hline Shreya Ghoshal & All \\
\hline Sonu Nigam & All \\
\hline Arijit Singh & Romantic, Happy, Sad \\
\hline Sunidhi Chauhan & Excited, Happy \\
\hline Kumar Sanu & Romantic, Happy \\
\hline Pankaj Udas & Sad, Happy, Trance \\
\hline Jagjit Singh & Sad, Trance \\
\hline Alka Yagnik & All \\
\hline Shaan Mukherjee & All \\
\hline Sukhvinder singh & Trance, Excited, Sad \\
\hline
\end{tabular}

Table 2 shows the mapping of singer with the moods based on the past performances made by them. There can be exception as the data shown in the table is generated considering only a small set of popular Hindi songs sung by the singers. After artist analysis the category of mood can be narrowed down to certain extent.

The tempo analysis can be done by adopting the approach of Matthew E. P. Davies and Mark D. Plumbley mentioned in their paper "Context-Dependent Beat Tracking of Musical Audio". They have used 2 state model where first state does the process of tempo induction and tracks tempo changes and second state maintains relative continuity within a single tempo assumption. [13]

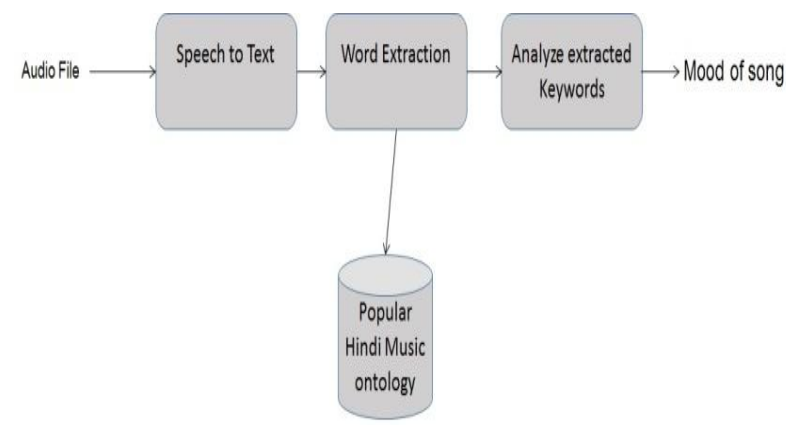

Fig 4: Lyrics analysis of Audio file

In case if more than one mood category at the end of tempo analysis, lyrics analysis module will be applied. This module has three sub modules as shown in fig 4 . The first module performs speech to text operation on the audio file received as input. The generated text is provided to the next module which is responsible for tokenizing the text and extracting words using the ontology for popular Hindi music. The third module will analyze the meaning of extracted words and finally tag a song according to the identified mood.

\section{CONCLUSION AND FUTURE WORK}

The model proposed in this paper presents the idea to generate mood based automatic play list for Hindi popular music. In this paper only limited set of moods have been considered but it can be extended for other mood categories in future. Further other assumptions such as genre to mood mapping and artist to mood mapping are based on limited set of popular songs. But larger data set needs to be analyzed for the accurate mappings. To automatically identify user's mood, the proposed model considers only widely used social platforms such as Facebook, Twitter and WhatsApp. Though to exactly find out user's mood, all social networking medium to which user is associated can be investigated. The correctness of tagging the song module highly depends on the algorithms used for speech to text conversion, Tempo analysis and created ontology for lyrics analysis.

\section{REFERENCES}

[1] Pohle, Tim, Elias Pampalk, and Gerhard Widmer. "Generating similarity-based playlists using traveling salesman algorithms." Proceedings of the 8th International Conference on Digital Audio Effects (DAFx-05). 2005.

[2] Van Zaanen, Menno, and Pieter Kanters. "Automatic Mood Classification Using TF* IDF Based on Lyrics." ISMIR. 2010.

[3] Goodman, Ron, and Howard N. Egan. "Automatic hierarchical categorization of music by metadata." U.S. Patent No. 6,928,433. 9 Aug. 2005.

[4] Brilis, Spyros, et al. "Mood classification using lyrics and audio: a case-study in Greek Music." Artificial Intelligence Applications and Innovations. Springer Berlin Heidelberg, 2012.

[5] Logan, Beth. "Content-Based Playlist Generation: Exploratory Experiments."ISMIR. 2002.

[6] Cunningham, Sally Jo, David Bainbridge, and Annette Falconer. "" More of an art than a science": Supporting the creation of playlists and mixes." (2006).

[7] Goto, Masataka, and Takayuki Goto. "Musicream: New Music Playback Interface for Streaming, Sticking, Sorting, and Recalling Musical Pieces."ISMIR. 2005.

[8] Liu, Dan, Lie Lu, and HongJiang Zhang. "Automatic mood detection from acoustic music data." ISMIR. 2003.

[9] Cardoso, Luís, Renato Panda, and Rui Pedro Paiva. "MOODetector: A prototype software tool for moodbased playlist generation." Simposio de InformaticaINForum 2011. Vol. 124. 2011.

[10] Davies, Matthew EP, and Mark D. Plumbley. "Contextdependent beat tracking of musical audio." Audio, Speech, and Language Processing, IEEE Transactions on 15.3 (2007): 1009-1020.

[11] Ujlambkar, Aniruddha M., and Vahida Z. Attar. "Automatic Mood Classification Model for Indian Popular Music." Modelling Symposium (AMS), 2012 Sixth Asia. IEEE, 2012.

[12] McKay, Cory, Ichiro Fujinaga, and Philippe Depalle. "jAudio: A feature extraction library." Proceedings of the International Conference on Music Information Retrieval. 2005. 
[13] Davies, Matthew EP, and Mark D. Plumbley. "Contextdependent beat tracking of musical audio." Audio, Speech, and Language Processing, IEEE Transactions on 15.3 (2007): 1009-1020.

[14] Ujlambkar, Amey, et al. "Mood Based Music Categorization System for Bollywood Music." International Journal of Advanced Computer Research 4.1 (2014): 223.

[15] Yang, Dan, and Won-Sook Lee. "Music emotion identification from lyrics."Multimedia, 2009. ISM'09. 11th IEEE International Symposium on. IEEE, 2009.
[16] Furuya, Mizuki, Hung-Hsuan HUANG, and Kyoji Kawagoe. "Evaluation of Music Classification Method based on Lyrics of English Songs." Proceedings of the International MultiConference of Engineers and Computer Scientists. Vol. 1. 2015.

[17] Hu, Xiao, and J. Stephen Downie. "When Lyrics Outperform Audio for Music Mood Classification: A Feature Analysis." ISMIR. 2010. 